\title{
Poor Compliance to Anti-Hypertensive Drugs in Saudi Arabia
}

\author{
Mohsen Jaber Yahya Alotayfi ${ }^{1}$, Salem Abdullah Alsohaimi ${ }^{1}$, Bushra Khalid AL-Qadi ${ }^{1}$, \\ Salma Mosa Kamil ${ }^{1}$, Alaa Jabril H Aththi ${ }^{1}$, Ghalia Alhussain Ahmed Alhazmi ${ }^{1}$, Halima \\ Ahmed Othman Qasem ${ }^{1}$, Amjad Ahmed Bugis ${ }^{2}$ \\ ${ }^{1}$ Jazan University, Saudi Arabia \\ ${ }^{2}$ Umm Al Qura University, Makkah, Saudi Arabia \\ *Corresponding Author: Mohsen Jaber Yahya Alotayfi, E-mail; mjyo545@hotmail.com
}

\begin{abstract}
Background: Poorly controlled hypertension may lead to several serious health problems, including stroke, aneurysms, coronary artery disease, kidney disease and peripheral artery disease.

Objectives: This study aimed at exploring the magnitude of the problem of non-compliance with antihypertensive drugs among patients in Saudi Arabia and identifying the associated factors.

Subjects and Methods: a cross-sectional study was carried out during the period January to August 2018 through social media sites including a sample of adult patients from different regions of the kingdom (Saudi and none-Saudi). Data were collected online through a questionnaire. The questionnaire included socio-demographic characteristics of hypertensive patients, history of smoking, and hypertension-related history. Adherence of patients to antihypertensive drugs was assessed utilizing an 8-item modified Morisky Medication Adherence Scale (8-MMMAS).

Results: The study included 267 hypertensive patients. About half of them (48.5\%) aged over 50 years. More than half were females $(58 \%)$, high educated $(62.3 \%)$ and employed $(46.7 \%)$. Approaching half of them, $42 \%$ has health insurance and 6 years or more of hypertension $(47.6 \%)$. About two-thirds of them $(68.8 \%)$ reported history of taking between one and three drugs/day and one dose of antihypertensive medication/day (64.8\%). High level of compliance with anti-hypertensive medications was observed among $6.2 \%$ of patients whereas medium and low levels were observed among $67.4 \%$ and $26.4 \%$ of them. High level of compliance was reported among patients aged over 50 years $(\mathrm{p}<0.001)$, females $(0.033)$, widowed $(0.001)$ and those haven'thistory of chronic diseases $(\mathrm{p}<0.001)$.
\end{abstract}

Conclusion: high compliance to anti-hypertensive medications in Saudi Arabia is low, particularly among male and young patients.

KEYWORDS: Anti-Hypertensive; Compliance; Saudi Arabia; Online

\section{INTRODUCTION}

The eighth report of the Joint National Committee on Prevention, Detection, Evaluation, and Treatment of High Blood Pressure (JNC - 8) recommended achieving blood pressure lower than $140 / 90 \mathrm{mmHg}$. or $130 / 80 \mathrm{mmHg}$ in hypertension without diabetes, or $<130 / 80 \mathrm{mmHg}$ in patients with diabetes and chronic kidney diseases ${ }^{(1)}$. Prevalence of hypertension among general population in western countries ranged between 28 and $44 \%{ }^{(2)}$, while its prevalence in Saudi Arabia ${ }^{(3)}$ was $26 \%$ and in other Arabic countries, it was $26.3 \%$ in Kuwait ${ }^{(4)}, 20.1 \%$ in $\underset{(7)}{\text { Egypt }}{ }^{(5)}, 33 \%$ in Oman ${ }^{(6)}$ and $32.1 \%$ in Qatar
Hypertension is a major risk factor for cardiovascular diseases morbidity and mortality ${ }^{(8)}$. Poorly controlled hypertension may lead to several serious health problems, including stroke, aneurysms, coronary artery disease, kidney disease and peripheral artery disease ${ }^{(9,10)}$. It has also a major economic impact ranging from medical costs to decrease in productivity ${ }^{(11,12)}$.

The treatment of hypertension depends on drug therapy and non- drug therapy, which mean life style modifications to decrease blood pressure and prevent its complications ${ }^{(13)}$. Low-compliance with anti- hypertensive medication puts patients at higher risk of cardiovascular events than high-compliant 
patients ${ }^{(14)}$. There are various factors affecting antihypertensive patient's compliance; patientrelated and non-patient related (factors related to physicians, disease characteristics and drugs) ${ }^{(15)}$.

Despite the dangerousness of uncontrolled hypertension, many patients are unaware that they have hypertension, and those known to be hypertensive are often not controlled ${ }^{(16,17)}$. This study aimed at exploring the magnitude of the problem of non- compliance with antihypertensive drugs among patients in Saudi Arabia and identifying the associated factors to put recommendations to avoid/control these problems.

\section{SUBJECTS AND METHODS}

A cross-sectional study was carried out online during the period January to August 2018 through social media sites including patients from different regions of the kingdom (Saudi and none-Saudi).

A representative sample of hypertensive adult patients (aged over 18 years), both genders, and all nationalities who have an access on any of social media were included in the study provided that he/she was on antihypertensive medication at the time of study conduction.

Sample size was computed utilizing online Roasoft sample size calculator with the assumption that the prevalence of non-perfect compliance with anti-hypertensive drugs of $72.1 \%$ (from previous local study) ${ }^{(18)}$, number of hypertensive patients with an online access exceeded 20000, level of confidence $95 \%$ and accepted error of $5 \%$. Thus, the minimum calculated sample size was 305 .

Data were collected online through a questionnaire. The questionnaire included socio-demographic characteristics of hypertensive patients (age, gender, educational level, job status, income and marital status), history of smoking, and hypertension- related history (having health insurance, duration of hypertension, history of co-morbid diseases, number of daily medications, types of antihypertensive, number of daily antihypertensive doses. Adherence of patients to anti-hypertensive drugs was assessed utilizing an 8-item modified Morisky Medication Adherence Scale (8-MMMAS). The scale was proved to be valid and reliable with sensitivity and specificity of $93 \%$ and $53 \%$, respectively ${ }^{(19)}$. Highly adherent patients were identified with a score of 8 on the scale, medium adherers with a score of 6 or $<8$, and low adherers with a score of $<6{ }^{(19)}$. The questionnaires were sent online to eligible participants on all social media sites (Facebook, Twitter, Instagram, etc...). A short statement explaining the objectives of the study was included before the questions.

Statistical Package for Social Sciences (SPSS) software version 23.0 was used for data entry and analysis. Descriptive statistics (number, percentage for categorical variables and mean, standard deviation and range for continuous variables) and analytic statistics using Chi Square tests $\left(\chi^{2}\right)$ to test for the association and/or the difference between two categorical variables were applied. P-value equal or less than 0.05 was considered statistically significant.

\section{RESULTS}

The study included 267 hypertensive patients. Their baseline information is summarized in table 1. About half of them (48.5\%) aged over 50 years. More than half were females $(58 \%)$, high educated $(62.3 \%)$ and employed $(46.7 \%)$. The income of more than one-third of them (36.2\%) ranged between 10001 and 20000 $\mathrm{SR} /$ month. Majority were married (77.6\%), not smoking $(72.1 \%)$ and have no chronic diseases $(81.9 \%)$. Approaching half of them (42\%) has health insurance and 6 years or more of hypertension (47.6\%). About two-thirds of them $(68.8 \%)$ reported history of taking between one and three drugs/day and one dose of antihypertensive medication/day (64.8\%).

High level of compliance with antihypertensive medications was observed among $6.2 \%$ of patients whereas medium and low levels were observed among $67.4 \%$ and $26.4 \%$ of them as illustrated in figure 1.

As shown in table 2, high level of compliance was reported among patients aged over 50 years $(\mathrm{p}<0.001)$, females $(0.033)$, widowed $(0.001)$ and those haven't history of chronic diseases $(\mathrm{p}<0.001)$. 
Table 1: Baseline information of the participants

\begin{tabular}{|c|c|c|c|}
\hline & & Frequency & Percentage \\
\hline \multirow[t]{4}{*}{ Age in years $(n=268)$} & $\leq 40$ & 64 & 23.9 \\
\hline & $41-45$ & 38 & 14.2 \\
\hline & 46-50 & 36 & 13.4 \\
\hline & $>\mathbf{5 0}$ & 130 & 48.5 \\
\hline \multirow[t]{2}{*}{ Gender $(n=278)$} & Male & 116 & 42.0 \\
\hline & Female & 160 & 58.0 \\
\hline \multirow[t]{3}{*}{ Educational level $(n=220)$} & Not educated & 6 & 2.7 \\
\hline & Secondary or less & 77 & 35.0 \\
\hline & Bachelor or above & 137 & 62.3 \\
\hline \multirow[t]{4}{*}{ Job status $(\mathrm{n}=268)$} & Employee & 125 & 46.7 \\
\hline & Unemployed & 97 & 36.2 \\
\hline & Retired & 36 & 13.4 \\
\hline & Others & 10 & 3.7 \\
\hline \multirow{4}{*}{ Income $(n=249)$} & $\leq \mathbf{5 0 0 0}$ & 64 & 25.7 \\
\hline & $5001-10000$ & 64 & 25.7 \\
\hline & $10001-20000$ & 90 & 36.2 \\
\hline & $>20000$ & 31 & 12.4 \\
\hline \multirow[t]{4}{*}{ Marital status } & Single & 23 & 8.3 \\
\hline & Married & 214 & 77.6 \\
\hline & Divorced & 11 & 4.0 \\
\hline & Widowed & 28 & 10.1 \\
\hline \multirow[t]{2}{*}{ Smoking } & Yes & 77 & 27.9 \\
\hline & No & 199 & 72.1 \\
\hline \multirow[t]{2}{*}{ Health insurance } & Yes & 116 & 42.0 \\
\hline & No & 160 & 58.0 \\
\hline \multirow[t]{3}{*}{ Duration of hypertension in years } & $1-3$ & 87 & 32.3 \\
\hline & $>3-<5$ & 54 & 20.1 \\
\hline & $\geq 6$ & 128 & 47.6 \\
\hline \multirow[t]{2}{*}{ History of chronic diseases } & Yes & 50 & 18.1 \\
\hline & No & 226 & 81.9 \\
\hline \multirow[t]{3}{*}{ Number of daily taken drugs $(n=260)$} & $1-3$ & 179 & 68.8 \\
\hline & $4-5$ & 35 & 13.5 \\
\hline & $>5$ & 46 & 17.7 \\
\hline \multirow[t]{3}{*}{ Number of antihypertensive doses $(n=256)$} & One & 166 & 64.8 \\
\hline & Two & 56 & 21.9 \\
\hline & Three & 34 & 13.3 \\
\hline
\end{tabular}

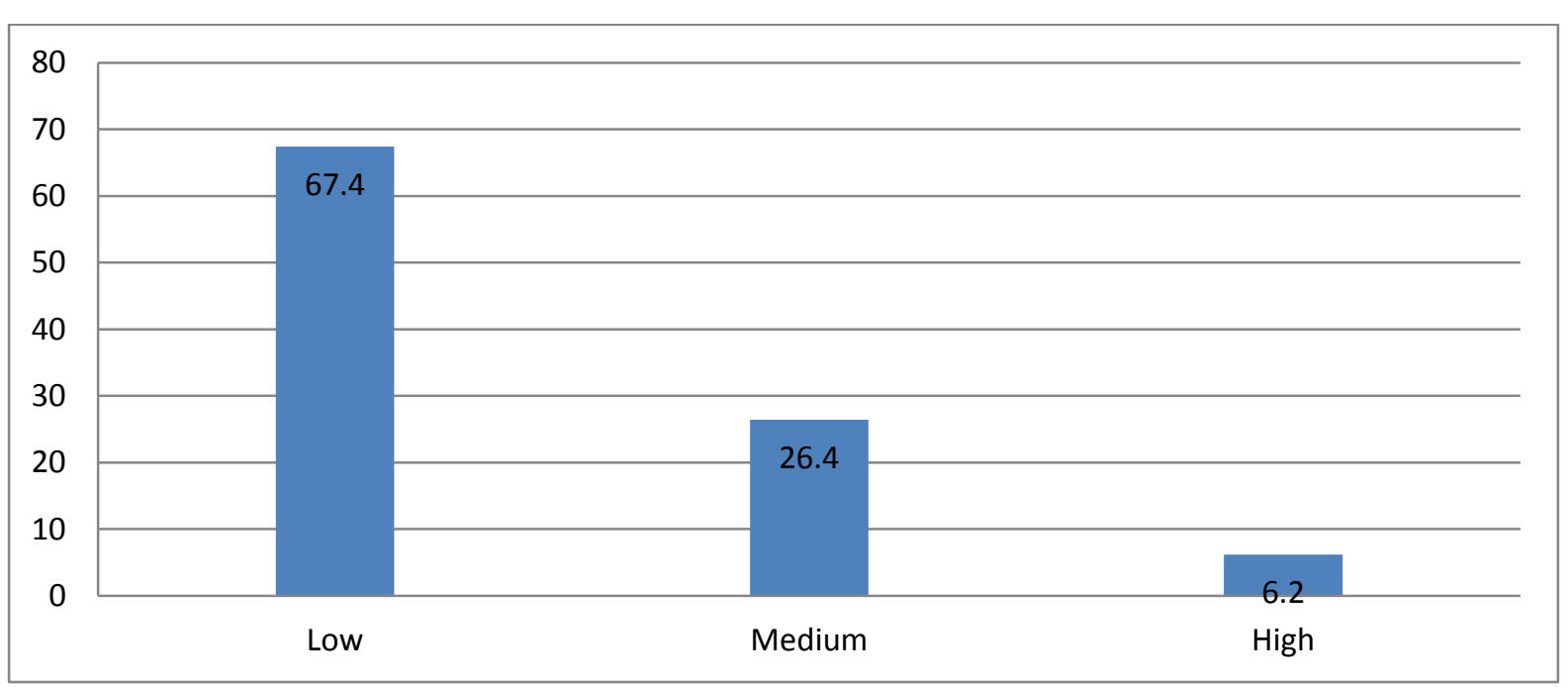

Fig 1: Level of compliance to anti-hypertensive medications among the participants 
Table 2: Factors contributing to compliance with anti-hypertensive medications among patients

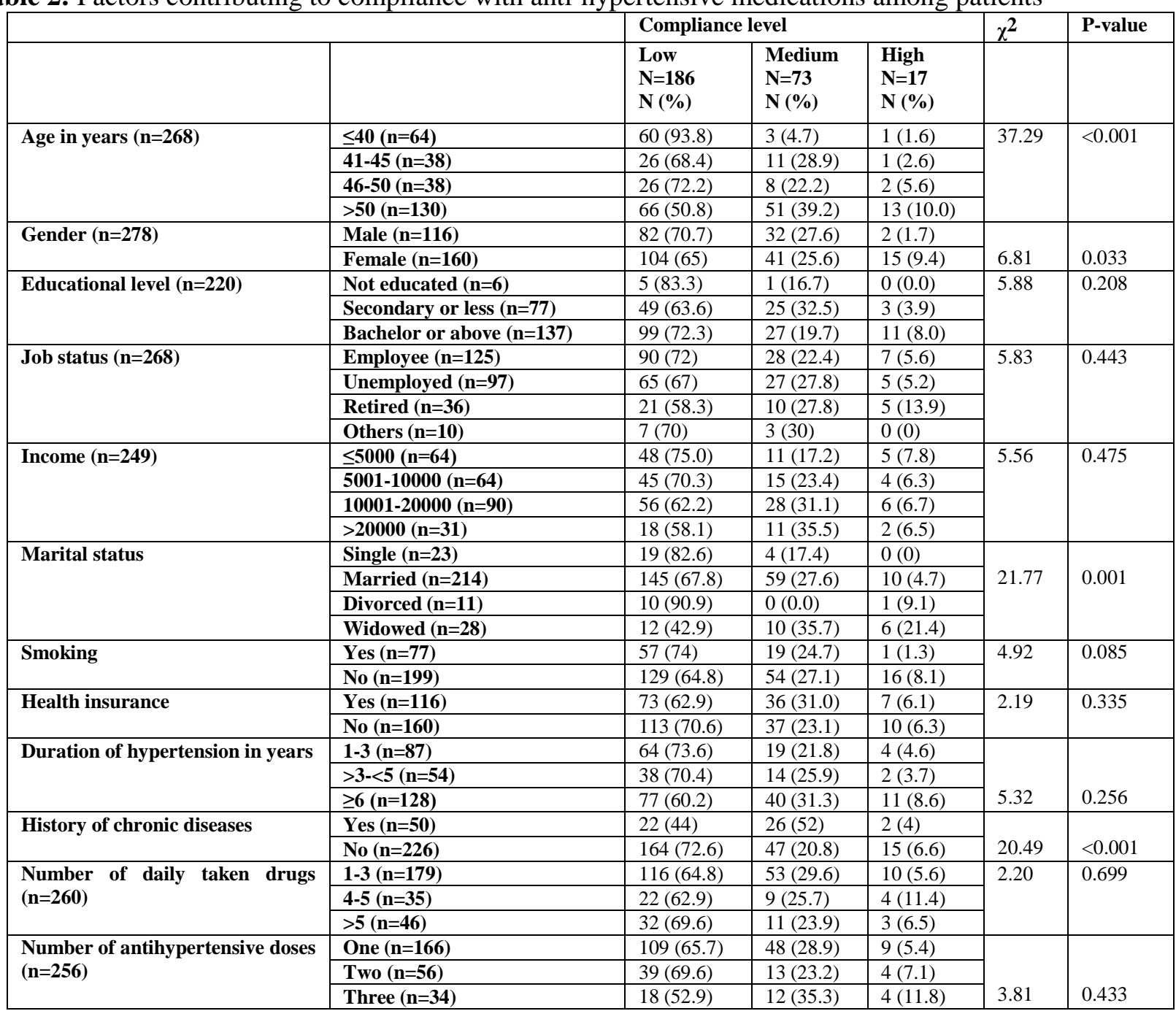

\section{DISCUSSION}

Among all other non-communicable diseases, hypertension has a specific importance because it is a major contributing factor for cardiovascular mortality ${ }^{(20)}$. Therefore, compliance of patients to treatment is essential in prevention of fatal cardiovascular diseases and poor compliance is associated with adverse outcomes and waste of resources ${ }^{(21)}$. This study aimed to assess the compliance of hypertensive patients in the Kingdom of Saudi Arabia to anti-hypertensive medications and identifying factors affecting adherence to these medications.

In the present study, the high compliance to anti-hypertensive was reported among only $6.2 \%$ of patients. This figure is extremely lower than those reported in another study carried out in Saudi Arabia (53\%) ${ }^{(21)}$, and other studies conducted in Lebanon $(50.5 \%)$ ${ }^{(20)}$, USA $(71.6 \%)^{(22)}$ and China $(65.1 \%)^{(23)}$. The extremely lower rate reported in the present study could be due to the fact that we depend in our analysis for definition of adherence on the consideration of high adherence of 8-MMMAS which identified with a score of 8 on the scale, however, most of other studies depend on a cut-off level of 6 out of 8. Also, the difference between our study and others could be due to different cultural factors. Regarding significant factors associated with high compliance; older (over 50 years), females, and widowed were more compliant with anti-hypertensive compared to others. These results are consistent with others who reported that younger patients and males were found to be less compliant ${ }^{(24)}$. However, older patients have been reported to be less compliant in other studies carried out in Tabuk, Saudi Arabia (21), Lebanon ${ }^{(20)}$, and Brazil $^{(25)}$.

Regarding medication-related factors, none of the studied factors in the current study was 
significantly associated with medication compliance. In studies carried out in Lebanon (20) and china (23), the number of antihypertensive medications was also not related to compliance behaviors. In France, it has been reported that taking a combination of antihypertensive drugs rather than multiple single drugs may improve compliance levels (26). Previous studies have shown that the relationship between dose frequency and low compliance is inconsistent as Claxton et al had reported that compliance rates decreased as the number of daily dose increased ${ }^{(27)}$, whereas, in contrast, Hashmi et al. ${ }^{(28)}$ had observed that adherence rates increased as the number of daily dose increased. However, in accordance with the present study, Inkster et al., found non-significant differences between level of adherence and daily dose frequency ${ }^{(29)}$.

Duration of hypertension was not associated with compliance with anti-hypertensive medications in the present study. This finding is inconsistent with what has been reported by Paul (2008) who observed that most chronic hypertensive patients have difficulty adhering to a prescribed regimen ${ }^{(30)}$.

It has been documented that patients with other chronic diseases may have to take more drugs that necessitate medication adherence (20). However, in the present study, having no comorbid diseases was significantly associated with high adherence to medications, which means that having only hypertension was associated with higher compliance to therapy. This study was not without limitations that should be considered. The design of the study as a cross-sectional one allowed testing association and not causation between compliance to antihypertensive drugs and associated factors. The non-inclusion of the level of blood pressure in order to associate between compliance with anti-hypertensive medications and blood pressure control is a limitation. The relationship between patients and their healthcare providers, which could affect their compliance with medications, was not included in the study. Finally, selfreporting questionnaire used as a method of collecting data in the present study has potential disadvantages regarding recall bias. The present study concluded that high compliance to anti- hypertensive medications in Saudi Arabia is low, particularly among male and young patients.
In the light of the study results, we recommended that health promotion and patient education programs as well as social support networks should be implemented in order to enhance compliance to drugs for hypertension.

\section{REFERENCES}

1. Thomas G, Shishehbor M, Brill D, Nally JV (2014): New hypertension guidelines: one size fits most? Cleve Clin J Med., 81(3): 178-88.

2. Wolf-Maier K, Cooper RS, Banegas JR, Giampaoli, Hense H, Joffres M (2003): Hypertension prevalence and blood pressure levels in 6 European countries, Canada and the United States. JAMA., 289: 2363-2369.

3. Al-Nozha MM, Abdullah M, Arafah MR, Khalil MZ, Khan NB,Al- Marou YY (2007): Hypertension in Saudi Arabia. Saudi Med J., 28: 77-84.

4. Al-Duwaisan HS, Al-Mhza AM, AlYaha AA, Al-Qattan MM (2003): Assessment of target organ damage in hypertension through a clinical audit in Kuwait family practice. Kuwait Med J., 35:202-207.

5. Ibrahim MM, Appel LJ, Rizk HH, Helmy S, Mosley J, Ashour Z (2001): Cardiovascular risk factors in normotensive and hypertensive Egyptians. J Hypertens., 19: 1933-1940.

6. Al-Riyami AA, Afifi MM (2002): Hypertension in Oman: distribution and correlates. J Egypt Public Health Assoc., 77:383-407.

7. Bener A, Al-Suwaidi J, AlJaber K, AlMarri S, Dogash MH, Elbagi IA (2004): The prevalence of hypertension and its associated risk factors in a newly developed country. Saudi Med J., 25: 918-922.

8. Wang $H$, Dwyer-Lindgren $L$, Lofgren KT, Rajaratnam JK, Marcus JR, Levin-Rector A et al. (2012): Agespecific and sex-specific mortality in 187 countries, 1970-2010: a systematic analysis for the Global Burden of Disease study 2010. Lancet, 380(9859): 2071-94.

9. Messerli FH, Williams B, Ritz E (2007): Essential hypertension. Lancet, 370(9587): 591-603.

10. He J, Whelton PK (1999): Elevated systolic blood pressure as a risk factor for cardiovascular and renal disease. Journal 
of Hypertension, 17(2): S7-S13.

11. Elliot WJ (2003): The economic impact of hypertension. The Journal of Clinical Hypertension, 5(2): 3-13.

12. Kearney PM, Whelton M, Reynolds K, Muntner P, Whelton PK, He J (2005): Global burden of hypertension: analysis of worldwide data. Lancet, 365(9455): 217 223.

13. Bell K, Twiggs J, Olin B (2015): Hypertension: The Silent Killer: Updated JNC-8 Guideline Recommendation, Available at: https://cdn.ymaws.com/www.aparx.org/resour ce/resmgr/CEs/CE_Hypertension_The Silent K.pdf

14. Mazzaglia G, Ambrosioni E, Alacqua M, Filippi A, Sessa E, Immordino V et al. (2009): Adherence to antihypertensive medications and cardiovascular morbidity among newly diagnosed hypertensive patients. Circulation, 120(16): 1598-605.

15. Turki AK, Sulaiman SAS (2009): Adherence to Antihypertensive Therapy in General Hospital of Penang: Does Daily Dose Frequency Matter? Jordan Journal of Pharmaceutical Sciences, 2(2): 167-176.

16. Al-Rukban MO, Al-Sughair AM, AlBader BO, Al-Tolaihi BA (2007): Management of hypertensive patients in primary health care setting: auditing the practice. Saudi Med J., 28: 85-90.

17. Al-Tuwijri AA, Al-Rukban MO (2006): Hypertension control and co- morbidities in primary health care centers in Riyadh. Ann Saudi Med., 26: 266-271.

18. Alsolami F, Correa-Velez I, Hou $X$ (2015): Factors affecting antihypertensive medications adherence among hypertensive patients in Saudi Arabia. American Journal of Medicine and Medical Sciences, 5(4): 181-189.

19. Morisky DE, Ang A, Krousel-Wood M, Ward HJ (2008): Predictive validity of a medication adherence measure in an outpatient setting. J Clin Hypertens (Greenwich), 10:348-54.

20. Yassine M, Al-Hajje A, Awada S, Rachidi S, Zein S, Bawab W et al. (2016): Evaluation of medication adherence in Lebanese hypertensive patients. J Epidemiol Glob Health, 6(3): 157-67.

21. Khalil SA, Elzubier AG (1997): Drug compliance among hypertensive patients in Tabuk, Saudi Arabia. J Hypertens., 15:561-5.

22. Hyre AD, Krousel-Wood MA, Muntner P, Kawasaki L, DeSalvo KB (2007): Prevalence and predictors of poor antihypertensive medication adherence in an urban health clinic setting. J Clin. Hypertensin (Greenwich), 9:179-86.

23. Lee GK, Wang HH, Liu KQ, Cheung Y, Morisky DE, Wong MC (2013): Determinants of medication adherence to antihypertensive medications among a Chinese population using Morisky Medication Adherence Scale. PLoS One, 8:e62775.

24. Marentette MA, Gerth WC, Billings DK, Zarnke KB (2002): Antihypertensive persistence and drug class. Can J Cardiol., 18:649-56.

25. Oliveira-Filho AD, Barreto-Filho JA, Neves SJ, Neves DP (2012): Association between the 8-item Morisky Medication Adherence Scale (MMAS-8) and blood pressure control. Arq Bras Cardiol., 99:649-58.

26. Korb-Savoldelli V， Gillaizeau F, Pouchot J, Lenain E, Postel- Vinay N, Plouin PF et al. (2012): Validation of a French version of the 8- item Morisky medication adherence scale in hypertensive adults. J Clin Hypertens (Greenwich), 14:429-34.

27. Claxton AJ, Cramer J , Pierce $\mathbf{C}$ (2001): A systematic review of the associations between dose regimens and medication compliance. Journal of Clinical Therapy, 3(2): 1296-1310.

28. Hashmi SK, Afridi MB, Abbas K, Sajwani RA, Saleheen D, Frossard PM, Ishaq M, Ambreen A, Ahmad U (2007): Factors associated with adherence to antihypertensive treatment in Pakistan. Journal of Pone, 2(3): 1310-1317.

29. Inkster ME, Donnan PT, MacDonald TM, Sullivan FM, Fahey $T$ (2006): Adherence to antihypertensive medication and association with patient and practice factors. Journal of Human Hypertensión, 20: 295- 297.

30. Paul S (2008): Poor adherence to oncedaily antihypertensive drugs is common. The New England Journal of Medicine, 1:25-31. 